\title{
L'ajustement dans l'application de la norme pour prendre en compte la conviction religieuse
}

Introduction

Vincente Fortier et Jean-Marie Woehrling

\section{CpenEdition}

\section{Journals}

Édition électronique

URL : http://journals.openedition.org/rdr/284

DOI : $10.4000 /$ rdr.284

ISSN : 2534-7462

Éditeur

Presses universitaires de Strasbourg

Édition imprimée

Date de publication : 1 mai 2019

Pagination : $9-12$

ISBN : 979-10-344-0045-4

ISSN : 2493-8637

\section{Référence électronique}

Vincente Fortier et Jean-Marie Woehrling, «L'ajustement dans l'application de la norme pour prendre en compte la conviction religieuse », Revue du droit des religions [En ligne], 7| 2019, mis en ligne le 25 novembre 2019, consulté le 21 novembre 2020. URL : http://journals.openedition.org/rdr/284 ; DOI : https://doi.org/10.4000/rdr.284

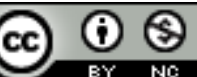

La revue du droit des religions est mise à disposition selon les termes de la Creative Commons Attribution - Pas d'Utilisation Commerciale 4.0 International - CC BY-NC 4.0. 


\section{INTRODUCTION L'AJUSTEMENT DANSL'APPLICATIONDE LA NORME POUR PRENDREEN COMPTE LACONVICTION RELIGIEUSE}

\section{Vincente FORTIER et Jean-Marie WOEHRLING}

Université de Strasbourg / CNRS, Droit, Religion, Entreprise et Société (DRES);

Président honoraire de tribunal administratif

— n précisant dans son arrêt du 22 novembre 2017 et à la suite de l'arrêt C de la Cour de justice de l'Union européenne «qu'il appartient à l'employeur de rechercher si, tout en tenant compte des contraintes inhérentes à l'entreprise et sans que celle-ci ait à subir une charge supplémentaire, il lui est possible de proposer à la salariée un poste de travail n'impliquant pas de contact visuel avec ces clients, plutôt que de procéder à son licenciement », la chambre sociale de la Cour de cassation n'a-t-elle pas ouvert la voie aux ajustements de la norme lorsque l'application de celle-ci au cas concret pose question au regard du respect d'une liberté (ici la liberté de religion), suscite un conflit de valeurs ou de normes, risque d'engendrer une discrimination?

C'est à l'articulation problématique de l'impératif juridique et de l'impératif religieux qu'est consacré ce dossier dont les trois premières contributions évoquent des questions de méthode pour résoudre les tensions, les trois suivantes étant dédiées à la réception de ces possibles méthodes dans trois pays européens, la France, la Belgique et l'Espagne. 
Qu'il s'agisse de travailler sur les techniques juridiques (accommodement, proportionnalité, concordance pratique) ou de procéder à l'analyse par État de l'implémentation de celles-ci, le point de vue des auteurs sur les ajustements de la norme positive aux convictions religieuses est distancié, leurs analyses nuancées et parfois désappointées!

À tout seigneur, tout honneur, le dossier s'ouvre avec les accommodements raisonnables, expression parfois abusivement utilisée et dont l'explicitation devait revenir à nos collègues québécois, Manon Montpetit et Stéphane Bernatchez. Corollaire du droit à l'égalité, l'obligation d'accommodement raisonnable est une construction essentiellement prétorienne afin d'assurer la mise en œuvre d'une «égalité réelle» plutôt que formelle. Manon Montpetit et Stéphane Bernatchez en exposent avec subtilité les soubassements, les arcanes du raisonnement, les bornes à son application. Et le lecteur découvrira, au fil des développements, que la simplicité de l'expression cache une évolution et une réalité complexes, sujettes à controverse.

En Europe, la problématique tenant à l'adaptation de la norme commune pour satisfaire la liberté de religion a également trouvé un écho à travers des concepts voisins ou distincts. Au premier chef, le principe de proportionnalité qui, selon les termes de Sébastien Van Drooghenbroeck et Xavier Delgrange, occupe une place cardinale dans le raisonnement juridique toutes les fois qu'il s'agit d'apprécier la licéité d'une action ou d'une abstention au regard des normes protectrices des droits et libertés fondamentaux. Comme l'accommodement raisonnable, le principe de proportionnalité est a priori d'une simplicité confondante dans sa compréhension et son maniement. Introduisant de la souplesse dans la règle de droit, en ce qu'il permet une application différenciée de celle-ci et respectueuse des droits fondamentaux, le principe de proportionnalité semble paré de toutes les vertus. Si l'analyse «macroscopique » à laquelle se livrent les auteurs met en lumière un système cohérent, en revanche une analyse plus fine, au microscope, en révèle les limites, «éclipses et angles morts».

Parmi les techniques juridiques permettant d'appréhender les conflits entre droits fondamentaux, on ne peut manquer d'évoquer la notion de «praktische Konkordanz » ou concordance pratique issue du droit constitutionnel allemand. Celle-ci insiste sur le fait que lorsque deux droits fondamentaux sont en conflit, aucun d'entre eux n'a vocation à se voir a priori sacrifié au profit de l'autre. Les deux droits en concurrence se doivent des concessions réciproques. Déclinant son étude sous les deux aspects de la liberté de religion - celle des organisations religieuses et celle des individus -, Bernhard Kresse 
montre que celle-ci fait l'objet d'une protection éminente en Allemagne. Nul besoin ici de déroger à la norme générale de droit civil: la mise en balance des intérêts antagonistes permet de résoudre les conflits de valeurs, «dans une concordance pratique des valeurs en cause».

Cette démarche d'ajustement ou d'aménagement de la norme au cas particulier soulève cependant aussi interrogations, résistances et critiques. Elle orienterait la gestion du droit vers des solutions particulières au cas par cas ou «sur mesure». Cette approche "casuistique» réduirait la sécurité juridique, elle pourrait conduire à un effritement de la norme commune, selon les termes de Frédéric Dieu, qui, dans sa contribution, met en regard le refus de principe des ajustements de la norme en France, où les principes constitutionnels de laïcité et d'égalité semblent s'opposer aux adaptations de la norme commune, et une jurisprudence tout en nuances réceptive à la satisfaction des demandes liées aux exigences religieuses.

À l'instar de Frédéric Dieu, Xavier Delgrange et Hélène Lerouxel font état des difficultés de principe à la «transplantation» de l'accommodement raisonnable en Belgique, ce pays étant aussi attaché que la France au culte de la loi et à l'égalité formelle. Toutefois, comme le soulignent les auteurs, la démocratie consociative belge qui favorise les accords contractuels avec les groupements, et partant le compromis et la négociation, permet la mise en place d'accommodements raisonnables. À partir d'exemples emblématiques de cette possibilité d'ajustement de la norme (clause de conscience, abattage rituel, maintien des écoles de confessions minoritaires, circoncision rituelle), Xavier Delgrange et Hélène Lerouxel mettent en lumière les inévitables tensions et contradictions qui traversent nos sociétés: si le chemin de l'égalité via les accommodements raisonnables est pavé de bonnes intentions, la route est encore longue et difficultueuse pour y parvenir.

Étudiant de manière très fine la doctrine de la Cour constitutionnelle espagnole, Javier Martínez-Torrón révèle ses évolutions parfois contradictoires et restrictives comme pour l'objection de conscience au service militaire, ou ses errements comme en matière de refus de transfusion sanguine par les Témoins de Jéhovah: parfois réceptive à la prééminence de la prescription religieuse, parfois à l'inverse, refusant la prise en charge par l'État d'un traitement alternatif à la transfusion de sang pratiqué dans une clinique privée. En dehors de la jurisprudence constitutionnelle, et pour ce qui concerne les relations de travail ou les symboles religieux dans l'espace public, Javier Martínez-Torrón note la même frilosité ou un manque de sensibilité à l'égard des ajustements de la norme pour satisfaire la liberté religieuse. En tout état de 
cause, l'auteur regrette dans certaines décisions l'absence de mise en balance des intérêts ou de mise en œuvre du principe de proportionnalité. Et pourtant, comme le note l'auteur, le besoin d'accommoder la norme commune aux exigences religieuses est fondamental dans des sociétés ouvertes, plurielles et devant faire toute leur place aux minorités religieuses.

Le thème de l'accommodement ou de l'ajustement soulève en effet des questions politiques et sociales sur le traitement des minorités et le choix des stratégies d'intégration. Les règles communes peuvent être «accommodées» pour faciliter leur participation dans le respect de leurs traditions propres. L'ajustement des normes prend ainsi place dans une politique de pluralisme culturel et de respect de la diversité.

Toutefois, l'accommodement ou l'ajustement ne constitue pas un droit absolu: toute personne ou groupe concerné de manière défavorable par une mesure générale ne peut prétendre à un accommodement. Quelle que soit la technique juridique utilisée ou la voie privilégiée, qui très souvent $\mathrm{du}$ reste se combinent, et même si le croyant ne saurait s'affranchir de la règle commune au nom de sa liberté de religion, l'effectivité de celle-ci et sa reconnaissance pleine et entière supposent des ajustements raisonnables du droit commun. À propos de l'affaire Leyla Sahin, la juge Tulkens a ainsi pu écrire dans son opinion dissidente: «Dans une société démocratique, je pense qu'il faut chercher à accorder - et non à opposer - les principes de laïcité, d'égalité et de liberté.»

À travers ces différentes contributions, le dossier opère une mise en abîme entre plusieurs droits fondamentaux: droit à l'égalité, principe de non-discrimination et droit à la liberté de religion, étroitement enchâssés, dans un rapport ambigu de répulsion et d'attirance. Il illustre également l'opposition parfois frontale entre système légaliste et mise en balance des intérêts. 